\title{
Biphasic Equilibrium Dialysis of Poly(N-Isopropyl Acrylamide) Nanogels Synthesized at Decreased Temperatures for Targeted Delivery of Thermosensitive Bioactives
}

\author{
Witold Musial, ${ }^{1}$ Jiri Michálek, ${ }^{2}$ Janusz Pluta, ${ }^{3}$ Anna Jaromin, \\ Magdalena Pietka-Ottlik, ${ }^{5}$ and Martin Pradny ${ }^{2}$ \\ ${ }^{1}$ Department of Physical Chemistry, Wroclaw Medical University, Borowska 211, 50-556 Wroclaw, Poland \\ ${ }^{2}$ Department of Polymer Gels, Institute of Macromolecular Chemistry of the Academy of Sciences of Czech Republic, \\ Heyrovského Nám. 2, 16206 Praha, Břevnov, Czech Republic \\ ${ }^{3}$ Department of Pharmaceutical Technology, Wroclaw Medical University, Borowska 211, 50-556 Wroclaw, Poland \\ ${ }^{4}$ Department of Lipids and Liposomes, Faculty of Biotechnology, University of Wroclaw, Przybyszewskiego 63/77, \\ 51-148 Wroclaw, Poland \\ ${ }^{5}$ Department of Organic Technology, Wroclaw University of Technology, Wybrzeze Wyspianskiego 27, 50-370 Wroclaw, Poland
}

Correspondence should be addressed to Witold Musial; witold.musial@gmail.com

Received 8 July 2013; Accepted 7 September 2013

Academic Editor: Kattimuttathu I. Suresh

Copyright (C) 2013 Witold Musial et al. This is an open access article distributed under the Creative Commons Attribution License, which permits unrestricted use, distribution, and reproduction in any medium, provided the original work is properly cited.

Hydrogel nanoparticles, referred to also as nanogels, are of special interest for medical and pharmaceutical applications. Due to small size in the range below the diameter of the capillaries, they are proposed as drug delivery carriers. The aim of the study was to estimate the influence of composition and reaction conditions during synthesis of poly-N-isopropyl acrylamide cross-linked by polyethylene glycol diacrylate on the purification rates of the polymer. Six types of thermosensitive nanogels were prepared by surfactant-free dispersion polymerization and assessed in terms of process yield, composition, and size at temperatures below and over volume phase temperature. During the diffusion of impurities, in the course of dialysis, assessed by the conductometric method, the remarkable influence of temperature and initiator concentration on the process was revealed. The release rates varied in the range between $9.63 \cdot 10^{-2}$ and $1.39 \cdot 10^{-1} \mathrm{~h}^{-1}$ in the first stage of the process, whereas in the second stage they were between $2.09 \cdot 10^{-2}$ and $6.28 \cdot 10^{-2} \mathrm{~h}^{-1}$. The evaluated time to obtain acceptable purity of the preparation was estimated to be in the range of 18 days. More detailed research should be directed towards the influence of the structure of obtained material on the purification process.

\section{Introduction}

Nanogels are of special interest for medical and pharmaceutical applications [1-3]. Due to small size in the range below the diameter of the capillaries, they are proposed as drug delivery carriers. One of the main problems is to overcome the impurity of the product, which is intended for peroral or parenteral applications. With the diameter range between 10 and $1000 \mathrm{~nm}$ severe complications arise, when synthesized entities undergo the purification process. Synthesized in different conditions, nanoparticles contain various amounts of residual organic solvents, monomers, comonomers, surfactants, inorganic salts, initiators, accelerators, and noncrosslinked polymer aggregates. The presence of these impurities should be kept at very low level, to avoid adverse reactions within the human organism. Usually, the nanoparticles are purified by dialysis with the use of semipermeable cellulose membranes. With time the rate in impurities diffusion through the membrane decreases to extremely low values, with parallel decrease of impurities concentration in donor solution retentate, during the process. For enhancement of that procedure, various approaches are evaluated, including concentration/diafiltration process membrane filtration, crossflow membrane filtration, filtration 
TABLE 1: The feed composition of the substrates, with respective assignments of the nanodispersions.

\begin{tabular}{|c|c|c|c|c|}
\hline \multirow{2}{*}{ Component } & \multirow{2}{*}{ Percentage [\%] } & \multicolumn{3}{|c|}{ Temperature of reaction $\left[{ }^{\circ} \mathrm{C}\right]$} \\
\hline & & 22 & 38 & 70 \\
\hline & & \multicolumn{3}{|c|}{ Assignments of the nanodispersions samples } \\
\hline TEMED & 0.01 & \multirow{4}{*}{ Nanodispersion 22-1 } & \multirow{4}{*}{ Nanodispersion 38-1 } & \multirow{4}{*}{ Nanodispersion $70-1$} \\
\hline APS & 0.02 & & & \\
\hline PEGDA & 0.25 & & & \\
\hline NIPA & 0.72 & & & \\
\hline TEMED & 0.02 & \multirow{4}{*}{ Nanodispersion 22-2 } & \multirow{4}{*}{ Nanodispersion $38-2$} & \multirow{4}{*}{ Nanodispersion $70-2$} \\
\hline APS & 0.04 & & & \\
\hline PEGDA & 0.25 & & & \\
\hline NIPA & 0.72 & & & \\
\hline
\end{tabular}

Abbreviations: in the text.

by electrospun nonwoven polymer fibers, purification by spin centrifugation dialysis, and many others [4-11].

Usually the large polymer aggregates may be eliminated by filtration through sintered glass filters. For the research needs, the dialysis using the concentration gradient is acceptable; however the onset of the "clean" product should be specified, that is, by conductometric measurements. For some materials the purification may last for really long time, for example, four weeks, whereas in some cases, the reasonable level of conductivity is obtained within one week [12-14].

Sensitive nanogels, according to their unique environmental responsiveness, have been widely applied in the fields of drug delivery carriers, tissue engineering, biological separation, and enzyme science [15]. Due to the characteristic temperature-responsive activity, the shape, volume, and the swelling ratio of poly- $\mathrm{N}$-isopropyl acrylamide (PNIPA) present specific changes upon reaching its volume phase transition temperature, which is around $32^{\circ} \mathrm{C}$ in aqueous solution. The polymer absorbs water to a swollen state at a temperature below the transition temperature and shrinks with volume decrease when the temperature increases above the VPTT (volume phase transition temperature). One of the new tasks within the forthcoming aspects of polymer science is synthesis of $\mathrm{N}$-isopropyl acrylamide derivatives in temperatures which do not exceed the temperature of proteins decomposition [16]. The formation of hydrogels as nano- or microstructures was evaluated by numerous authors, whereas Dušek developed in details the problem of so called microsyneresis; in the case of $\mathrm{N}$-isopropyl acrylamide increased temperature plays important role in microgel formation [17-19]. The aim of the study was to estimate the influence of composition and reaction conditions on the estimated dialysis rates. Six nanogels were prepared by surfactant-free dispersion polymerization and assessed in terms of process yield, composition, and size. Within the synthesis process the $\mathrm{N}, \mathrm{N}, \mathrm{N}^{\prime}, \mathrm{N}^{\prime}$-tetramethylethane-1,2-diamine (TEMED) was used to accelerate the free radical polymerization process [20]. During the diffusion of impurities, assessed by the conductometric method, the influence of temperature and initiator concentration on the purification process was evaluated. The purification rates were calculated assuming first order release of impurities from the donor compartment retentate, to the acceptor compartment permeate. Additionally the evaluated time to obtain acceptable purity of the preparation was estimated for the poly-N-isopropyl acrylamide-co-polyoxyethylene glycol diacrylate (PNIPA-co-PEGDA) polymer.

\section{Material and Methods}

2.1. Materials. N-isopropyl acrylamide (NIPA), polyethylene glycol diacrylate of molecular mass ca. 2000 (PEGDA), and $\mathrm{N}, \mathrm{N}, \mathrm{N}^{\prime}, \mathrm{N}^{\prime}$-tetramethyl-ethane-1,2-diamine were supplied by Aldrich (Czech Republic, Prague). Ammonium persulfate (APS) was purchased from Lachema (Czech Republic, Prague). The components obtained from industrial suppliers were used without further purification. A dialysis bag with a molecular weight cut-off of 12000-14000 Da was obtained from Sigma Aldrich (Czech Republic, Prague). Deionized water was obtained from the TKA DI 6000 system (Germany, Niederelbert) and was used in all the following procedures.

2.2. Synthesis of the Thermosensitive Nanoparticles. PNIPA nanogel particles crosslinked by PEGDA were synthesized by surfactant-free dispersion polymerization (SFDP) in deionized water under an inert nitrogen atmosphere according to the procedure evaluated by Pelton and Chibante [21, 22] and developed in the group of Lowe et al., and in other groups [23-25]. The radical initiator, ammonium persulfate, was placed in a $500 \mathrm{~mL}$, three-necked round-bottomed flask and stirred continuously at $120 \mathrm{~m}^{-1}$. Predissolved NIPA and cross-linking agent PEGDA were dissolved in $20 \mathrm{~mL}$ of deionized water with stirring and were then added to the reaction vessel. After $4 \mathrm{hrs}$, the dispersion was cooled to room temperature and filtered. Dry weight analysis of the nanogels showed the dispersion to have a concentration on the order of $0.1-0.5 \mathrm{wt} . \%$. The starting composition of the substrates is given in Table 1, with respective assignments of the nanodispersions.

2.3. Equilibrium Dialysis Purification. Dialysis-purification by diffusion through semipermeable membrane was performed in the set of glass vessels. The sample of $50 \mathrm{~mL}$ of 
TABLE 2: The yields of nanogels synthesized in various temperature conditions and different concentrations of initiator.

\begin{tabular}{lccc}
\hline & \multicolumn{3}{c}{ Yield [\%] } \\
Concentration of initiator [\%] & 0.02 & 0.04 \\
\hline \multirow{2}{*}{ Temperature $\left[{ }^{\circ} \mathrm{C}\right]$} & 22 & 8 & 16 \\
& 38 & 24 & 89 \\
& 70 & 76 & 93 \\
\hline
\end{tabular}

nanogel dispersion was closed in dialysis bag and transferred into $300 \mathrm{~mL}$ glass vessel. The system was stirred in IKAVIBRAX-VXR device (Germany), by $100 \mathrm{rpm}$. The particles were dialyzed against deionized water until the conductivity was less than $1.0 \mu \mathrm{S} \cdot \mathrm{cm}^{-1}$ [26]. Within the first $30 \mathrm{hrs}$ the conductivity in the acceptor compartment was measured, and the water remained unchanged in the acceptor compartment. After $30 \mathrm{hrs}$, the water was replaced in acceptor compartment by fresh deionized water, and the conductivity measurement was taken after $24 \mathrm{hrs}$. The procedure was repeated every $24 \mathrm{hrs}$, through 18 days, till the conductivity did not exceed $1.0 \mu \mathrm{S} \cdot \mathrm{cm}^{-1}$.

2.4. FTIR Evaluations. Fourier transformed infrared spectra of the obtained polymers were measured at ambient temperature with a Nicolet Nexus 870 FTIR spectrometer purged with dry air and equipped with a Golden Gate device ATR, with reproducible approximate sample loads of $3 \mathrm{kbar}$. Samples were measured in the dry state; the spectra were corrected for absorption of the solvent and $\mathrm{H}_{2} \mathrm{O}$ vapors.

2.5. Assessment of the Hydrodynamic Diameter. The hydrodynamic diameter was assessed in aqueous dispersions by dynamic light scattering (DLS). From the ca. $50 \mathrm{~mL}$ sample, $50 \mu \mathrm{L}$ was taken and centrifugated within the Eppendorf tube filled with $1.0 \mathrm{~mL}$ of prefiltered water, in Roth MicroCentrifuge, at $6000 \mathrm{rpm}$ for $45 \mathrm{~min}$. From the tube $50 \mu \mathrm{L}$ of supernatant were taken and placed into polystyrene-single use fluorimeter cuvettes. Then $1 \mathrm{~mL}$ of deionized water, filtered through $0.2 \mu \mathrm{m}$ PVDF Whatman nanofilter, was added to the cuvette and the sample was inserted into Zetasizer Nano with $173^{\circ}$ backscatter measurement arrangement, and settings of Mark-Houwink parameters. The duration of measurements was extended for the case of large particles, with relaxation time multiplier of 100000 . The measurements settings were automated to seek optimum position; the attenuator selection was also automated.

2.6. Evaluation of the Conductivity Data from Purification Experiment. We assumed that the observed process of the release of impurities from the donor compartment may be considered as the first order process. The calculations were performed using obtained data, assuming one-stage process of the release of impurities and two-stage process of the release of impurities. The fitted functions of one-stage, first order kinetics model and two-stage, first order kinetics model were compared by using the correlation factors $\left(r^{2}\right)$. The assumption was made that the conductivity parameter

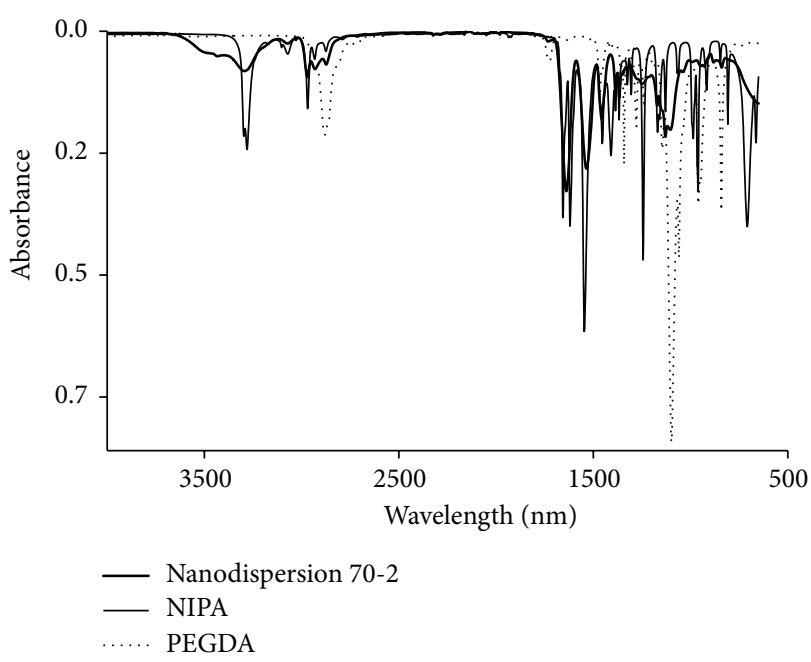

FIGURE 1: The FTIR spectra of N-isopropyl acrylamide (NIPA), polyethylene glycol diacrylate of molecular mass ca. 2000 (PEGDA), and freeze-dried Nanodispersion 70-2.

reflects the concentration of impurities within the diluted nanodispersions.

\section{Results and Discussion}

Due to the FTIR assessments within all the polymeric entities, the PNIPA polymer was synthesized in all the cases with implemented PEGDA cross-linker-on Figure 1, there is exemplification of the spectrum of NIPA, PEGDA, and freeze-dried nanodispersion.

The yield of the reaction varied, depending on the applied conditions and compositions. In general, the yield of nanogel, calculated on the basis of gravimetric assessment, was in the range between $8 \%$ and $93 \%$. The yields for nanogel are presented in Table 2.

Within the first $30 \mathrm{hrs}$ of the purification process, the conductivity was measured every $1 \mathrm{hr}$. The conductivity in all the assessed cases decreased in the donor compartment with time, and course of the obtained function adhered, with high correlation factors, to the logarithmic function, which reflected the first order reaction, as it is presented on (1), where $C$ and $C_{0}$ are the actual conductivity in the donor compartment and initial conductivity in the donor compartment, respectively. The $k$ and $t$ denominate the process rate and the actual time:

$$
C=C_{0} \cdot e^{-k t} .
$$

This fact supports the idea of first order process diffusion which is triggered by the concentration gradient in the assessed copolymers. However we observed some variety between the evaluated nanogels, in terms of measured conductivity. For quantitative evaluation of the results, we treated the obtained data by the method usually applied for the release experiments. Due to the experimental data, the course of the conductivity increases and can be divided into two stages. In the first stage the increasing is faster than in the 
TABLE 3: Correlation factors $\left(r^{2}\right)$ calculated for course of the function time versus conductivity, assuming 1-stage or 2-stage release of impurities from the donor compartment, during purification process of synthesized nanogels, where the $A$ and $B$ are the theoretically evaluated starting values of conductivity, which reflect the initial concentrations for the first and for the second stage, with respective $\alpha$ and $\beta$ rates of the process [28].

\begin{tabular}{|c|c|c|c|c|}
\hline Model type & & 1 stage & & 2 stages \\
\hline Equation & & $C=C_{0} \cdot e^{-k t}$ & & $C=A \cdot e^{-\alpha t}+B \cdot e^{-\beta t}$ \\
\hline \multirow{12}{*}{ Preparation } & \multirow{2}{*}{ Nanodispersion 22-1 } & \multirow{2}{*}{0.8413} & 1 & 0.9736 \\
\hline & & & 2 & 0.9944 \\
\hline & \multirow{2}{*}{ Nanodispersion 38-1 } & \multirow{2}{*}{0.8554} & 1 & 0.9799 \\
\hline & & & 2 & 0.9930 \\
\hline & \multirow{2}{*}{ Nanodispersion 70-1 } & \multirow{2}{*}{0.8277} & 1 & 0.9696 \\
\hline & & & 2 & 0.9893 \\
\hline & \multirow{2}{*}{ Nanodispersion 22-2 } & \multirow{2}{*}{0.9383} & 1 & 0.9921 \\
\hline & & & 2 & 0.9886 \\
\hline & \multirow{2}{*}{ Nanodispersion 38-2 } & \multirow{2}{*}{0.8797} & 1 & 0.9795 \\
\hline & & & 2 & 0.9797 \\
\hline & \multirow{2}{*}{ Nanodispersion 70-2 } & \multirow{2}{*}{0.7202} & 1 & 0.9489 \\
\hline & & & 2 & 0.9585 \\
\hline
\end{tabular}

second stage. Higher values of correlation factors confirmed the 2-stage course idea. In Table 3 the correlation factors for the 1-stage and 2-stage models are presented, according to equations developed in earlier work [28].

Due to the experimental data, the two-stage process seems to be more probable than the one-stage. The data presented in Figure 2 depict the course of conductivity increasing in the acceptor compartment. The upper straight lines on the graph represent the upper limit of the conductivity of the nanodispersion, measured in parallel samples: after 30 hrs, but before purification process-this value reflects the highest content of impurities in the sample. The values of conductivity in the first stage of the purification were in agreement with the simple predictions. In the case of high initiator level, the donor compartment released faster impurities than in the case of lower initiator level in the reactor. Also the level of conductivity was higher, compared to that of nanogels synthesized with low feed content of initiator-Figure 2.

The maximal level, due to the prediction equation within the first step of the release of impurities, should be obtained after ca. $14 \mathrm{hrs}, 42 \mathrm{hrs}$, and $48 \mathrm{hrs}$ for the nanogels 70-1, 381 , and 22-1, respectively, as it is assigned by dashed circles in Figure 2(a). Increased concentration of initiator in the feed composition influenced the level of conductivity during the purification but we did not identify any obvious tendency in the process. The estimated times of final equilibrium, due to the estimations from the first step equation, are between 23 and 28 hrs. From the data gathered in Table 3, as it was mentioned, the course of the impurities release investigated by the conductivity measurements should be bi-phasic. The second stage of conductivity increase is presented in Figure 3, both for the low and high feed content of the initiator in the reacting mixture.

The evaluation of the second stage of the purification reveals that the 70-1 sample will be finally equilibrated after almost 100 hrs, whereas the sample 70-2, with double amount
TABLE 4: The rates of the increase in the conductivity, within the acceptor compartment calculated for course of the function of time versus conductivity, assuming two-stage release of impurities from the donor compartment, during purification process of synthesized nanogels.

\begin{tabular}{lcc}
\hline Rate in & 1st stage $\left[\mathrm{h}^{-1}\right]$ & 2nd stage $\left[\mathrm{h}^{-1}\right]$ \\
\hline Nanodispersion 22-1 & $9.63 \cdot 10^{-2}$ & $2.65 \cdot 10^{-2}$ \\
Nanodispersion 38-1 & $1.03 \cdot 10^{-1}$ & $2.95 \cdot 10^{-2}$ \\
Nanodispersion 70-1 & $1.25 \cdot 10^{-1}$ & $3.28 \cdot 10^{-2}$ \\
Nanodispersion 22-2 & $1.39 \cdot 10^{-1}$ & $6.28 \cdot 10^{-2}$ \\
Nanodispersion 38-2 & $1.26 \cdot 10^{-1}$ & $4.21 \cdot 10^{-2}$ \\
Nanodispersion 70-2 & $1.27 \cdot 10^{-1}$ & $2.09 \cdot 10^{-2}$ \\
\hline
\end{tabular}

of initiator in the feed composition, will not reach equilibrium even after double time of, that is, $200 \mathrm{hrs}$. Oppositely, the samples 22-2 and 38-2 should reach the equilibrium just after around 50 hrs, whereas the samples 22-1 and 38-1 will be still in the course of dialysis due to the equation, describing the second stage of conductivity increase. The calculated rates are gathered in Table 4.

From the main course of the purification process in first $30 \mathrm{hrs}$ it may be seen that the release of impurities from the donor compartment is faster in the first stage of the process, and one raw slower, after ca. $10 \mathrm{hrs}$. The increase in temperature of the reacting mixture, in the case of low feed content of initiator, resulted in faster release of impurities in the purification procedure. This tendency was observed both in the first and in the second stage of the purification process as it is presented in Table 4. However the rates of release of the impurities, measured by the conductivity phenomena, were ordered actually in a decreasing way, when the high amount of initiator was applied. The kinetics of impurities release was measured in the period of first $30 \mathrm{hrs}$, without change of the water. Then, the data were collected in $24 \mathrm{hr}$ intervals, 


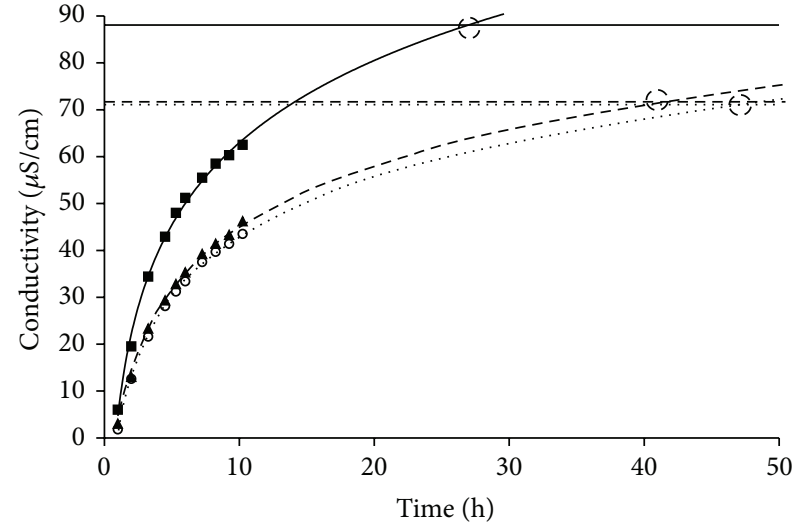

- Nanodispersion 70-1 DP

\ Nanodispersion 38-1 DP

o Nanodispersion 22-1 DP

(a)

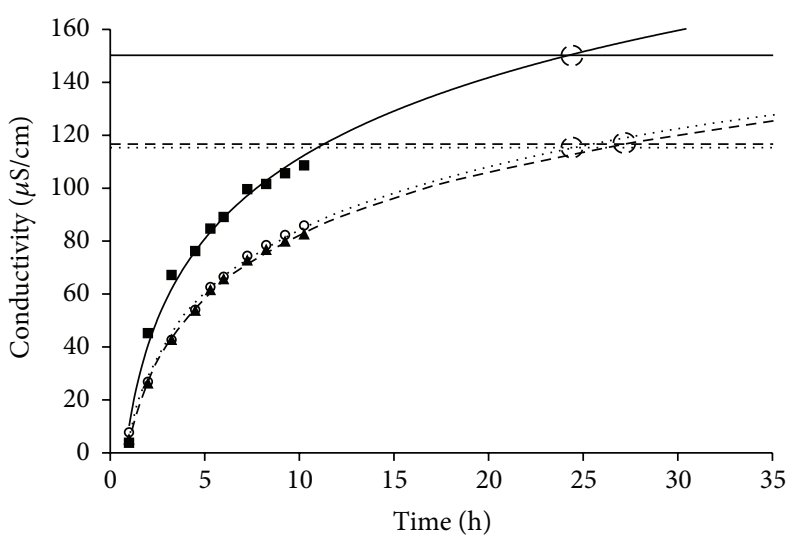

- Nanodispersion 70-2 DP — Nanodispersion 70-2 BP

\ Nanodispersion 38-2 DP - - - Nanodispersion 38-2 BP

○ Nanodispersion 22-2 DP …. Nanodispersion 22-2 BP

(b)

FIGURE 2: The first stage of purification of aqueous dispersion of a nanogel, measured in terms of conductivity, for nanogels synthesized with low (a) and high (b) feed content of initiator. The dashed circles represent the intersection of the conductivity curve with a straight line which represents the upper possible level of conductivity in specified samples. DP refers to the data from the purification process in acceptor compartment-the lines are the guides for the eye. BP refers to the data of the same concentration and volume, not purified-straight lines, explanation in the text. Other acronyms which are given in Table 3 refer to the feed composition of the nanogels.

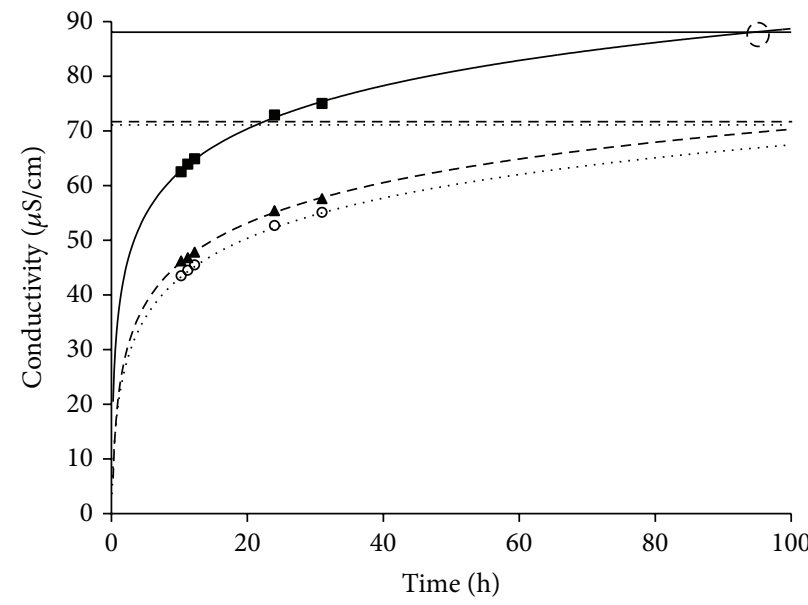

- Nanodispersion 70-1 DP — Nanodispersion 70-1 BP

\ Nanodispersion 38-1 DP - - - Nanodispersion 38-1 BP

o Nanodispersion 22-1 DP …. Nanodispersion 22-1 BP

(a)

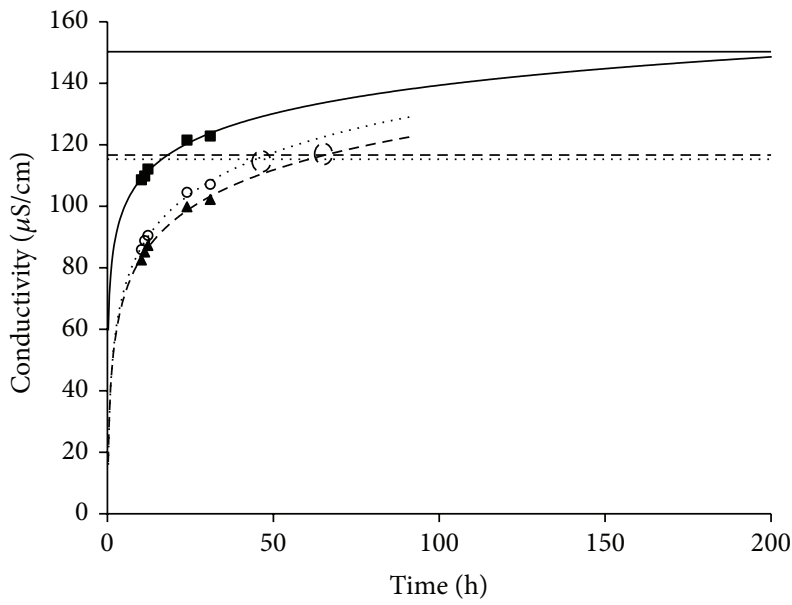

- Nanodispersion 70-2 DP — Nanodispersion 70-2 BP

\ Nanodispersion 38-2 DP - - Nanodispersion 38-2 BP

o Nanodispersion 22-2 DP ..... Nanodispersion 22-2 BP

FIGURE 3: The second stage of purification of aqueous dispersion of a nanogel, measured in the terms of conductivity, for nanogels synthesized with low (a) and high (b) feed content of initiator. The dashed circles represent the intersection of the conductivity curve with a straight line which represents the upper possible level of conductivity in specified samples. DP (during purification) refers to the data from the purification process in acceptor compartment-the lines are the guides for the eye. BP (before purification) refers to the data of the same concentration and volume, not purified-straight lines, explanation in the text. Other acronyms which are given in Table 3 refer to the feed composition of the nanogels.

preceded by change of the water in acceptor compartment. The data are gathered in Figure 4.

The complete purification of the nanogel dispersion was confirmed after the eighth to the tenth day, with final values close to $1.0 \mu \mathrm{S} \cdot \mathrm{cm}^{-1}$. However, after keeping the dispersion in isolated environment, the conductivity increased in acceptor compartment to ca. $5 \mu \mathrm{S} \cdot \mathrm{cm}^{-1}$, but after consequent dialysis purification through four days it decreased to the value of $1.0 \mu \mathrm{S} \cdot \mathrm{cm}^{-1}$ and maintained for prolonged period. As the synthesized nanogels are intended for drug delivery, with the 


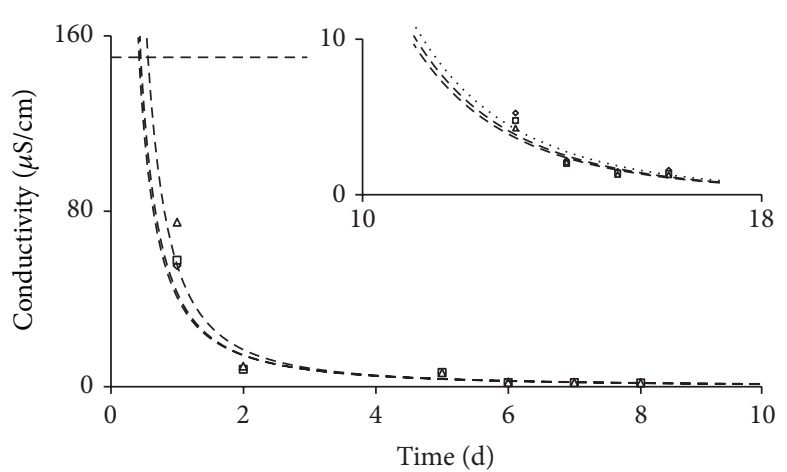

(a)

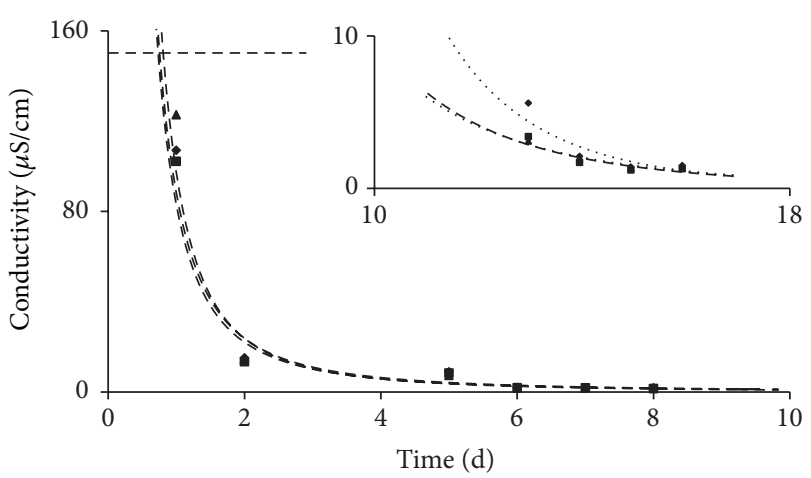

(b)

FIgURE 4: The conductivity in acceptor compartment, after $24 \mathrm{hr}$ period of static dialysis by deionized water. The assignments are as follows: nanodispersion 22-1 $(\diamond)$, nanodispersion 38-1 $(\square)$, nanodispersion 70-1 $(\triangle)$, nanodispersion 22-2 ( $)$, nanodispersion 38-2 ( $\bullet)$, and nanodispersion 70-2 ( $\mathbf{\Delta})$, with straight line on the large graph representing the initial conductivity in the donor compartment. The inserted small graphs give information on the conductivity after the tenth to the eighteenth day of static dialysis.

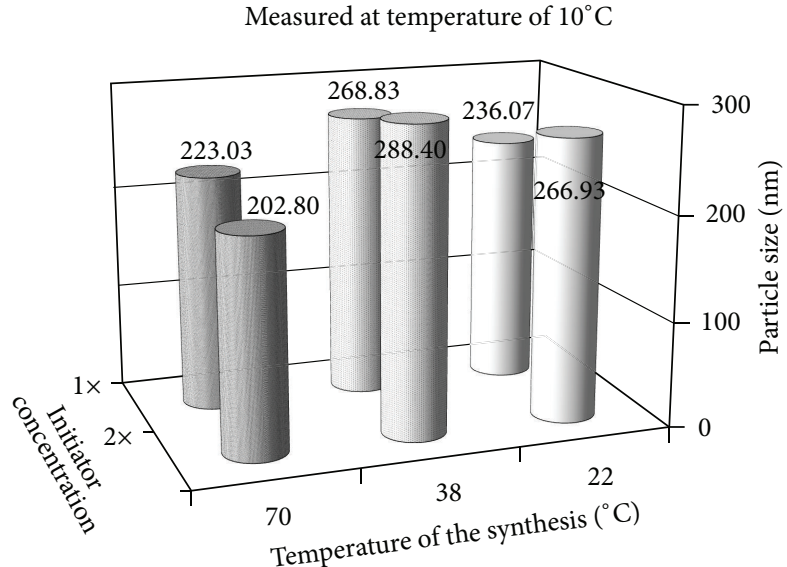

(a)

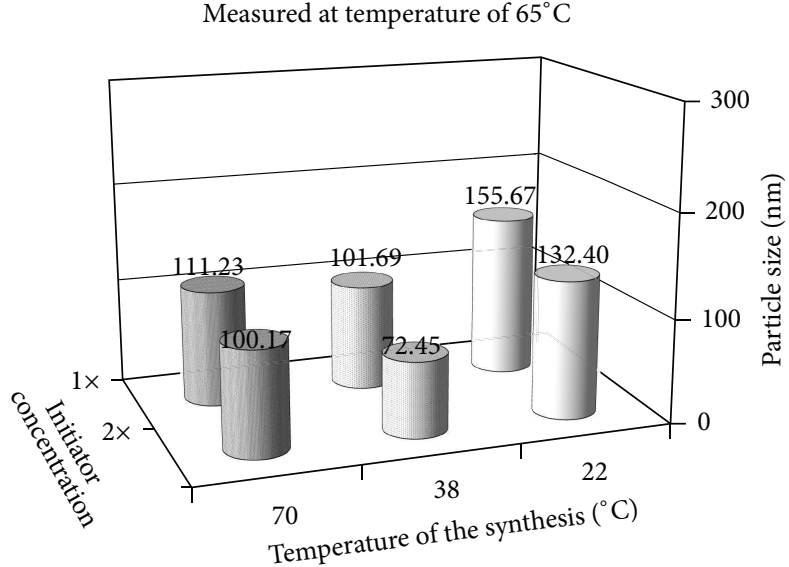

(b)

Figure 5: The hydrodynamic diameters, measured by DLS at temperatures of $10^{\circ} \mathrm{C}$ and $65^{\circ} \mathrm{C}$. The concentration of the initiator (Init. conc.) was varied in experiments between $0.01 \%(1 \mathrm{x})$ and $0.02 \%(2 \mathrm{x})$. Particle size refers here to the hydrodynamic diameter.

trigger factor, temperature, the most interesting issue is the thermosensitivity of obtained structures. The hydrodynamic diameters measured in the DLS device are in the range between $72.45 \mathrm{~nm}$ and $155.67 \mathrm{~nm}$ when measured at $65^{\circ} \mathrm{C}$; however the hydrodynamic diameter is much higher, when the assay is performed at decreased temperature of $10^{\circ} \mathrm{C}$, that is, between $202.80 \mathrm{~nm}$ and $288.40 \mathrm{~nm}$. We gathered the data within the graph in Figure 5, with respect to the concentration of the initiator and to the temperature of the synthesis.

Obtained yield values presented in Table 2 indicate that, with the increase in the temperature in the course of the reaction polymerization, the initiator seems to be more active in the system. The applied concentration of accelerator TEMED seems to be acceptable for the initial growth of the polymer molecules; however at decreased temperatures also the polymerization rate seems to decrease. The elevated level of the main initiator APS favors the reaction course. The data from DLS measurements performed at elevated temperatures suggest that the application of increased amount of initiator leads to the increase in number of particles in the system, what is in agreement with observations of Chiu and Lee $[29,30]$ as well as with some theoretical predictions [31, 32].

The differences in dialysis course are connected to the remains of the unreacted components within the reaction mixture. However, the results obtained in the conductometric measurements may indicate also the influence of product structure on the course of release of the impurities gathered in the nanogel. According to the development of small-angle neutron scattering (SANS) technique the evaluation of the substructures of microgels became more feasible [33]. The concept of two compartments in the structure of microgels and nanogels, derivatives of $\mathrm{N}$-isopropylacrylamide, was initially developed by $\mathrm{Wu}$ et al.; due to theirs observations, the monomers distribution was more dense within the center of the synthesized microgels [34]. This idea was confirmed by 


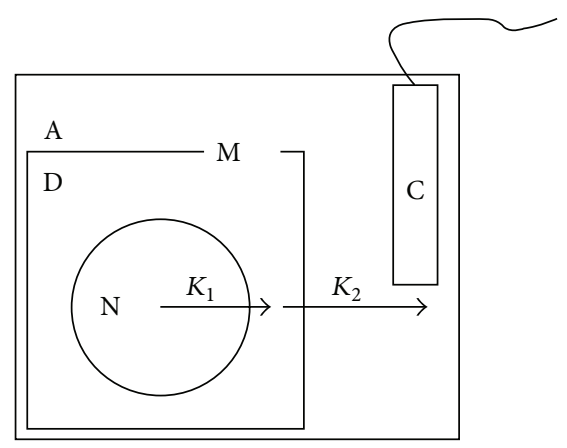

(a)

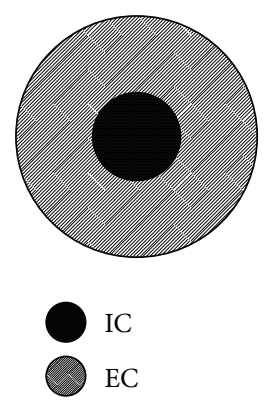

(b)

FIGURE 6: The scheme of dialysis purification of obtained nanogels (a) and schematic representation of two compartmental microgel structures (b), where $\mathrm{N}$ is the nanogel, $\mathrm{D}$ is the donor compartment with retentate, $\mathrm{A}$ is the acceptor compartment with permeate, $\mathrm{M}$ is the semipermeable membrane of MWCO $12-14 \mathrm{kDa}, \mathrm{C}$ is the conductivity probe, $K_{1}$ is the diffusion rate of impurities from the internal nanogel structure, $K_{2}$ is the diffusion rate of impurities from the solution into the donor compartment, IC is the internal compartment and EC is the external compartment of the nanogel due to the evaluation in reference [27].

other researchers in optical measurements [35], as well as in several SANS studies [36-38]. The detailed evaluation of the topic was given later by Sanders-there is still open question, if the internal and external compartments of the microgel origin from ongoing synthesis or following agglomeration and cross-linking [27]. Due to the fact that almost $90 \%$ of the microgel in water consists of water, the soluted impurities should be distributed nonhomogenously within the polymeric matrix [39]. Partially the impurities may be distributed within the core, and partially within the shell which is less dense [40]. We suggest in our case two-step purification process, due to the scheme presented in Figure 6(a). In the first phase the purification course is dominated by the diffusion of the impurities from the external water phase, that is, $K_{2}>K_{1}$, which reflects the impurities in the aqueous environment and the external compartment of the nanogel. During the second phase the process is ruled mainly by the diffusion of the impurities from the internal nanogel phase, internal compartment, and $K_{1}>K_{2}$. The respective values for theoretical estimation of the acceptable purification time may be red from the graphs in Figure 3. The initial level of impurities was in the highest in the case of nanodispersion
70-2, and the time for sufficient purification is the longest. The relatively short time for acceptable purification course is estimated in the case of nanodispersion 38-2 and nanodispersion 22-2. nanodispersion 70-1, nanodispersion 381 , and nanodispersion 22-1 may be characterized by similar, rather long purification rate. As it was mentioned above, the important factors affecting the impurities release from the nanoparticles may include both the level of reactants, and the internal structure of the nanogel. To evaluate the topic we calculated the rates of impurities release from the donor compartment, implementing the idea of two-stage and first order kinetics. The calculations confirm the idea of influence of structure on the purification time of the nanodispersions. In the first stage the impurities release is comparable within the group of low starting values of initiator: $9.63 \cdot 10^{-2}, 1.03$. $10^{-1}$, and $1.25 \cdot 10^{-1} \mathrm{~h}^{-1}$. Also in the group of nanodispersions produced with high amount of initiator, the purification rates calculated on the basis of conductometric assays are similar: $1.39 \cdot 10^{-1}, 1.26 \cdot 10^{-1}$, and $1.27 \cdot 10^{-1} \mathrm{~h}^{-1}$. However in the second stage of the purification process, the rates increased for samples prepared with low initiator concentration, with the temperature of reaction. Oppositely the rates in the second stage decreased in samples prepared with higher amount of initiator-Table 4. By high initiator concentration, at elevated temperatures higher amount of components was used to form polymer particles. When low concentration of initiator was applied the structures of nanoparticles were characterized by higher size-at elevated temperature the particles were able to preserve high amount of impurities, which were released with lag time from the core-shell nanostructures, as it is presented in Figure 6(b). Evaluation of the impurities release from the bicompartmental microgel structure in the terms of bi-phasic kinetic process should have remarkable impact on the future experiments, for example, in the field of controlled drug release. From practical point of view the acceptable purity is in our case acquired after 18 days of dialysis through semipermable membrane.

\section{Conclusion}

Both, composition and reaction, conditions influence the acceptable time for purification of nanogel dispersions obtained with $\mathrm{N}$-isopropyl acrylamide-co-polyethylene glycol diacrylate. The purification course may be divided into two kinetic stages. At low concentrations of initiator the residual impurities seem to be at lower level. When high concentration of initiator is applied, the respective impurity level is higher and the purification time should be elapsed. After 18 days of the process of purification, the nanogel dispersions were cleaned within acceptable level of conductivity, not higher than $1.0 \mu \mathrm{S} \cdot \mathrm{cm}^{-1}$. More detailed research should be directed towards the influence of the structure of obtained material on the purification process, as the bicompartmental structure of the nanogel may be crucial for the process. The optimal dialysis time for the synthesized nanoparticles was estimated to be 18 days. 


\section{Conflict of Interests}

None of the authors has any conflict of interests to disclose in this work.

\section{Acknowledgment}

The study was supported by research fellowship within the "Development Program of Wroclaw Medical University" funded by the European Social Fund, Human Capital, National Cohesion Strategy, Contract no. UDA-POKL. 04.01.01-00-010/08-00.

\section{References}

[1] J. K. Oh, D. I. Lee, and J. M. Park, "Biopolymer-based microgels/nanogels for drug delivery applications," Progress in Polymer Science, vol. 34, no. 12, pp. 1261-1282, 2009.

[2] J. K. Oh, R. Drumright, D. J. Siegwart, and K. Matyjaszewski, "The development of microgels/nanogels for drug delivery applications," Progress in Polymer Science, vol. 33, no. 4, pp. 448477, 2008.

[3] J. K. Oh, S. A. Bencherif, and K. Matyjaszewski, "Atom transfer radical polymerization in inverse miniemulsion: a versatile route toward preparation and functionalization of microgels/nanogels for targeted drug delivery applications," Polymer, vol. 50, no. 19, pp. 4407-4423, 2009.

[4] I. Limayem, C. Charcosset, and H. Fessi, "Purification of nanoparticle suspensions by a concentration/diafiltration process," Separation and Purification Technology, vol. 38, no. 1, pp. $1-9,2004$.

[5] D. Jassby, S.-R. Chae, Z. Hendren, and M. Wiesner, "Membrane filtration of fullerene nanoparticle suspensions: effects of derivatization, pressure, electrolyte species and concentration," Journal of Colloid and Interface Science, vol. 346, no. 2, pp. 296302, 2010

[6] S. Kim, M. Marion, B.-H. Jeong, and E. M. V. Hoek, "Crossflow membrane filtration of interacting nanoparticle suspensions," Journal of Membrane Science, vol. 284, no. 1-2, pp. 361-372, 2006.

[7] K. M. Yun, C. J. Hogan Jr., Y. Matsubayashi, M. Kawabe, F. Iskandar, and K. Okuyama, "Nanoparticle filtration by electrospun polymer fibers," Chemical Engineering Science, vol. 62, no. 17, pp. 4751-4759, 2007.

[8] R. Mihaila, S. Chang, A. T. Wei et al., "Lipid nanoparticle purification by Spin Centrifugation-Dialysis (SCD): a facile and high-throughput approach for small scale preparation of siRNA-lipid complexes," International Journal of Pharmaceutics, vol. 420, no. 1, pp. 118-121, 2011.

[9] Z. Ding, G. Chen, and A. S. Hoffman, "Synthesis and purification of thermally sensitive oligomer-enzyme conjugates of poly(n-isopropylacrylamide)-trypsin," Bioconjugate Chemistry, vol. 7, no. 1, pp. 121-126, 1996.

[10] J. Gao and B. J. Frisken, "Cross-linker-free N-isopropylacrylamide gel nanospheres," Langmuir, vol. 19, no. 13, pp. 5212-5216, 2003.

[11] T. Gilányi, I. Varga, R. Mészáros, G. Filipcsei, and M. Zrínyi, "Interaction of monodisperse poly(N-isopropylacrylamide) microgel particles with sodium dodecyl sulfate in aqueous solution," Langmuir, vol. 17, no. 16, pp. 4764-4769, 2001.

[12] M. M. Yallapu, J. K. Vasir, T. K. Jain, S. Vijayaraghavalu, and V. Labhasetwar, "Synthesis, characterization and antiproliferative activity of rapamycin-loaded poly( $\mathrm{N}$-isopropylacrylamide)based nanogels in vascular smooth muscle cells," Journal of Biomedical Nanotechnology, vol. 4, no. 1, pp. 16-24, 2008.

[13] N. Singh and L. A. Lyon, "Au nanoparticle templated synthesis of pNIPAm nanogels," Chemistry of Materials, vol. 19, no. 4, pp. 719-726, 2007.

[14] H. Ayame, N. Morimoto, and K. Akiyoshi, "Self-assembled cationic nanogels for intracellular protein delivery," Bioconjugate Chemistry, vol. 19, no. 4, pp. 882-890, 2008.

[15] X. He, S. Yu, Y. Dong, F. Yan, and L. Chen, "Preparation and properties of a novel thermo-responsive poly $(\mathrm{N}$ isopropylacrylamide) hydrogel containing glycyrrhetinic acid," Journal of Materials Science, vol. 44, no. 15, pp. 4078-4086, 2009.

[16] A. Qin, M. Lu, Q. Liu, and P. Zhang, "Synthesis and characterization of thermo-sensitive poly (N-isopropylacrylamide) hydrogel with fast response rate," Frontiers of Chemistry in China, vol. 2, no. 2, pp. 135-139, 2007.

[17] K. Dušek, "Phase separation during the formation of threedimensional polymers," Journal of Polymer Science C, vol. 16, pp. 1289-1299, 1967.

[18] K. Dušek, "Quasichemical equilibrium approach to crosslinked polymer solutions," Journal of Polymer Science C, vol. 39, no. 1, pp. 83-106, 1972.

[19] K. Dušek, "Network build-up and structure in curing of epoxy resins," Makromolekulare Chemie. Macromolecular Symposia, vol. 7, no. 1, pp. 37-53, 1987.

[20] J. I. Ngadaonye, M. O. Cloonan, L. M. Geever, and C. L. Higginbotham, "Synthesis and characterisation of thermosensitive terpolymer hydrogels for drug delivery applications," Journal of Polymer Research, vol. 18, no. 6, pp. 2307-2324, 2011.

[21] R. H. Pelton and P. Chibante, "Preparation of aqueous latices with N-isopropylacrylamide," Colloids and Surfaces, vol. 20, no. 3, pp. 247-256, 1986.

[22] R. Pelton, "Temperature-sensitive aqueous microgels," Advances in Colloid and Interface Science, vol. 85, no. 1, pp. 1-33, 2000.

[23] J. S. Lowe, B. Z. Chowdhry, J. R. Parsonage, and M. J. Snowden, "The preparation and physico-chemical properties of poly $(\mathrm{N}$ ethylacrylamide) microgels," Polymer, vol. 39, no. 5, pp. 1207$1212,1998$.

[24] H. Nur, V. T. Pinkrah, J. C. Mitchell, L. S. Benée, and M. J. Snowden, "Synthesis and properties of polyelectrolyte microgel particles," Advances in Colloid and Interface Science, vol. 158, no. 1-2, pp. 15-20, 2010.

[25] M. Rasmusson, A. Routh, and B. Vincent, "Flocculation of microgel particles with sodium chloride and sodium polystyrene sulfonate as a function of temperature," Langmuir, vol. 20, no. 9, pp. 3536-3542, 2004.

[26] H. Senff and W. Richtering, "Influence of cross-link density on rheological properties of temperature-sensitive microgel suspensions," Colloid and Polymer Science, vol. 278, no. 9, pp. 830-840, 2000.

[27] B. R. Saunders, N. Laajam, E. Daly, S. Teow, X. Hu, and R. Stepto, "Microgels: from responsive polymer colloids to biomaterials," Advances in Colloid and Interface Science, vol. 147-148, pp. 251262, 2009.

[28] A. A. Kubis, W. Musial, and M. Szczesniak, "Influence of some polysorbates on hydrocortisone release from hydrophilic gels considered as two-compartment models," Pharmazie, vol. 57, no. 7, pp. 479-481, 2002. 
[29] Y. Y. Chiu and L. J. Lee, "Microgel formation in the free radical crosslinking polymerization of ethylene glycol dimethacrylate (EGDMA). I. Experimental," Journal of Polymer Science A, vol. 33, no. 2, pp. 257-267, 1995.

[30] Y. Y. Chiu and L. J. Lee, "Microgel formation in the free radical crosslinking polymerization of ethylene glycol dimethacrylate (EGDMA). II. Simulation," Journal of Polymer Science A, vol. 33, no. 2, pp. 269-283, 1995.

[31] K. Dusek, "Formation-structure relationships in polymer networks," British Polymer Journal, vol. 17, no. 2, pp. 185-189, 1985.

[32] K. Dušek, "Formation process, structure, and properties of polymer networks," in Polymer Networks, pp. 1-6, VSP, Utrecht, The Netherlands, 1992.

[33] I. Berndt, J. S. Pedersen, and W. Richtering, "Structure of multiresponsive "intelligent" core-shell microgels," Journal of the American Chemical Society, vol. 127, no. 26, pp. 9372-9373, 2005.

[34] X. Wu, R. H. Pelton, A. E. Hamielec, D. R. Woods, and W. McPhee, "The kinetics of poly(N-isopropylacrylamide) microgel latex formation," Colloid \& Polymer Science, vol. 272, no. 4, pp. 467-477, 1994.

[35] I. Varga, T. Gilányi, R. Mészáros, G. Filipcsei, and M. Zrínyi, "Effect of cross-link density on the internal structure of poly(Nisopropylacrylamide) microgels," Journal of Physical Chemistry B, vol. 105, no. 38, pp. 9071-9076, 2001.

[36] B. R. Saunders, "On the structure of poly(N-isopropylacrylamide) microgel particles," Langmuir, vol. 20, no. 10, pp. 39253932, 2004.

[37] T. G. Mason and M. Y. Lin, "Density profiles of temperaturesensitive microgel particles," Physical Review E, vol. 71, no. 4, Article ID 040801, 2005.

[38] M. Stieger, J. S. Pedersen, P. Lindner, and W. Richtering, "Are thermoresponsive microgels model systems for concentrated colloidal suspensions? A rheology and small-angle neutron scattering study," Langmuir, vol. 20, no. 17, pp. 7283-7292, 2004.

[39] C. Wu and X. Wang, "Globule-to-coil transition of a single homopolymer chain in solution," Physical Review Letters, vol. 80, no. 18, pp. 4092-4094, 1998.

[40] I. Berndt, J. S. Pedersen, and W. Richtering, "Temperaturesensitive core-shell microgel particles with dense shell," Angewandte Chemie - International Edition, vol. 45, no. 11, pp. 17371741, 2006. 

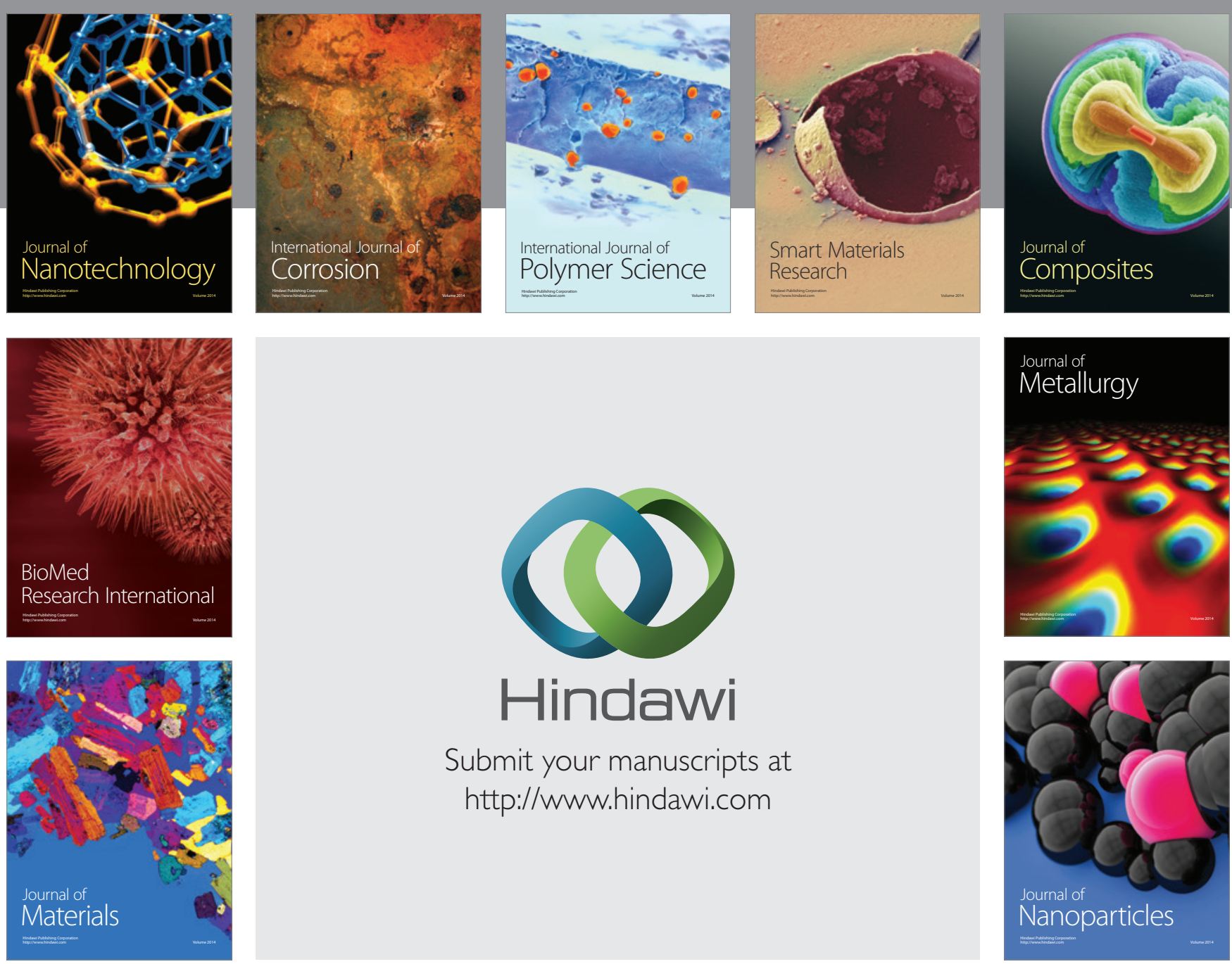

Submit your manuscripts at http://www.hindawi.com
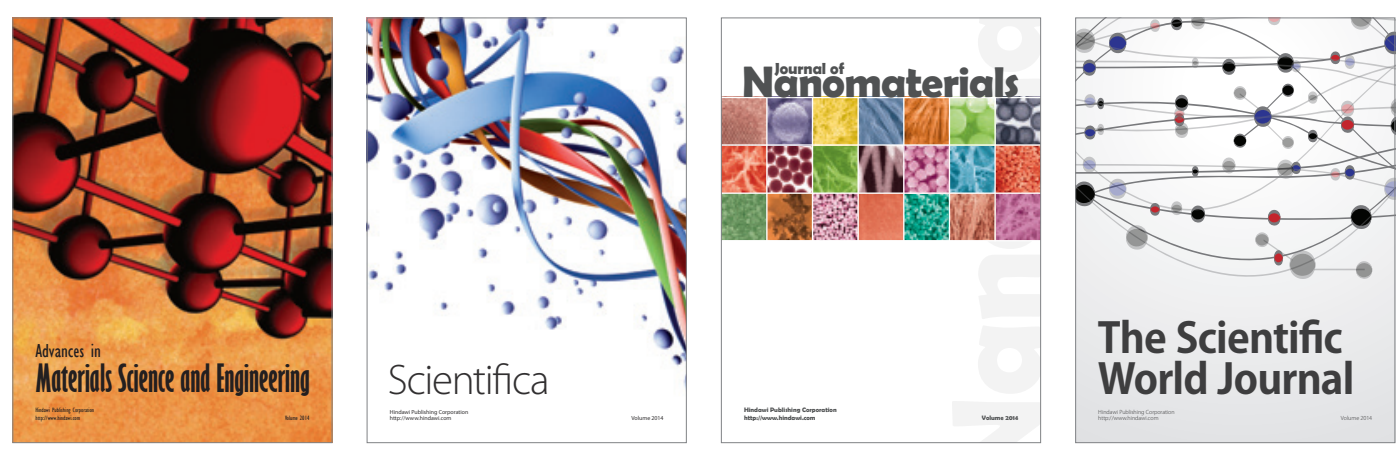

\section{The Scientific World Journal}
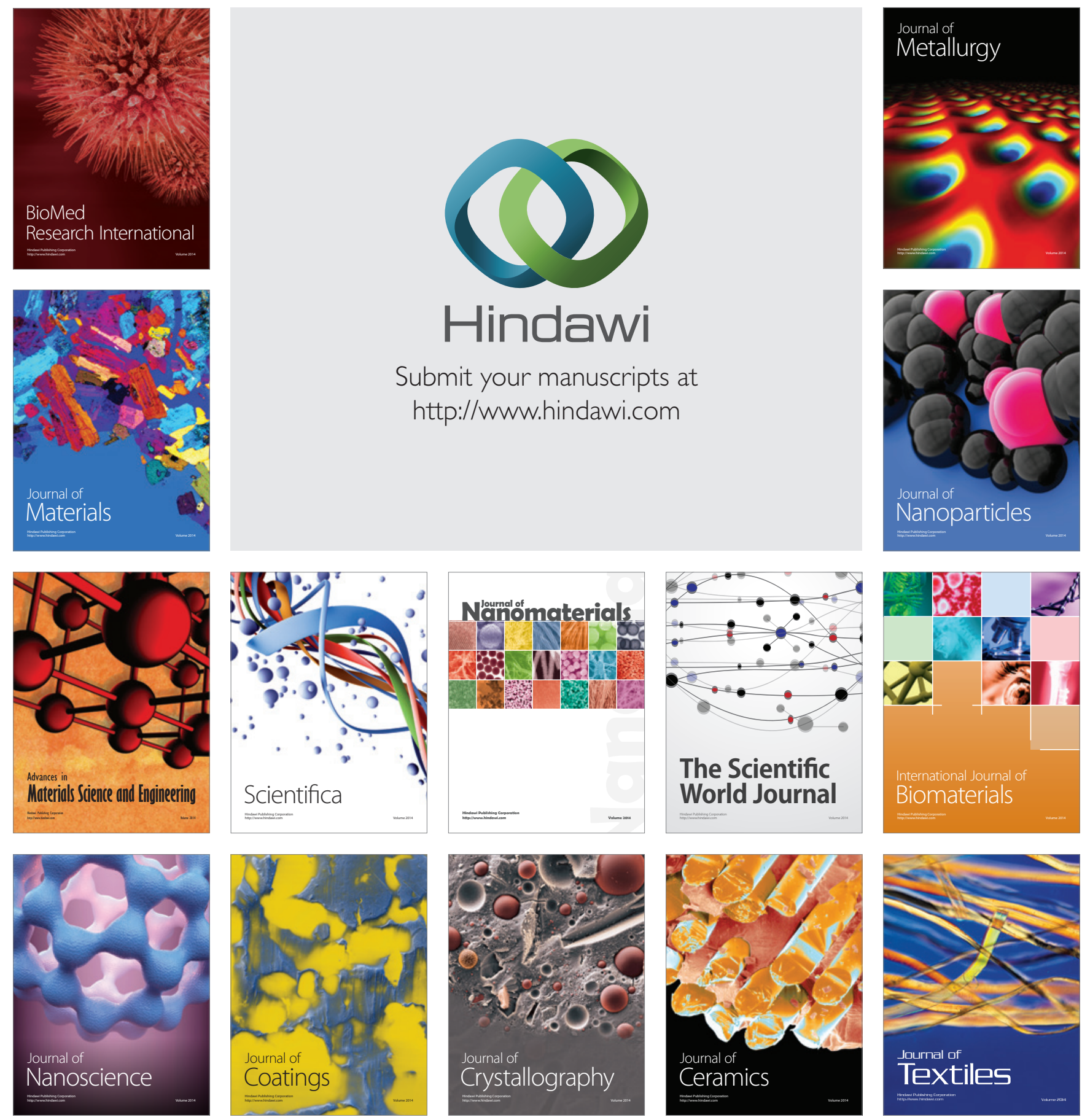